\title{
Digoxin increased risk of death in women, but not men, with heart failure
}

\author{
Rathore SS, Wang Y,Krumholz HM. Sex-based differences in the effect of digoxin for the treatment of heart failure. $N$ Engl \\ J Med 2002;347:1403-11.
}

\section{QUESTION: Does the effect of digoxin therapy differ in men and women with heart failure (HF) and depressed left ventricular systolic function?}

\section{Design}

Randomised \{allocation concealed $\}^{*}$, blinded (\{participants, healthcare providers, $\} \dagger$ and data collectors), placebo controlled trial with up to 48 months of follow up.

\section{Setting}

302 clinical centres in the US and Canada.

\section{Patients}

5281 men (median age 64 y, $87 \%$ white, $30 \%$ New York Heart Association [NYHA] class $\geq \mathrm{III}$ ) and 1519 women (median age $66 \mathrm{y}, 81 \%$ white, $40.6 \%$ NYHA class $\geq \mathrm{III}$ ) who had clinically confirmed HF (ie, current or past clinical symptoms or signs or radiographic evidence of pulmonary congestion) and an ejection fraction $\leq 45 \%$. \{Follow up was $99 \%$. $\}^{*}$

\section{Intervention}

2642 men and 755 women were allocated to digoxin, and 2639 men and 764 women were allocated to placebo. The initial recommended dose was based on a nomogram that accounted for age, sex, weight, and renal function $(0.125-0.500 \mathrm{mg} / \mathrm{d})$.

\section{Main outcome measures}

All cause mortality. Secondary outcome measures included death from all cardiovascular causes, death from worsening HF, hospital admission for worsening

Source of funding:

National Heart, Lung.

and Blood Institute.

For correspondence no information available. mortality or cardiovascular death; women in the digoxin group had an increased risk of all cause mortality and cardiovascular death (table). Digoxin reduced death and hospital admission caused by worsening HF in men but not women (table). More men in the digoxin group than the placebo group had hospital admissions for other causes; in women, the groups did not differ (table).

\section{Conclusion}

Digoxin led to an increased risk of all cause and cardiovascular death in women, but not in men, with heart failure and depressed left ventricular systolic function.

*The Digitalis Investigation Group. $N$ Engl J Med 1997;336:525-33.

$\dagger$ Information provided by author.

\section{COMMENTARY}

A systematic review of the effectiveness of digitalis glycosides in the treatment of $\mathrm{HF}$ in patients with normal sinus rhythm concluded that mortality did not differ between treatment and control groups, while patients treated with digitalis had a lower rate of hospital admission and clinical deterioration. ${ }^{1}$ What was missing from the studies reviewed was any exploration of possible sex differences in response to treatment.

Rathore et al addressed that gap through secondary analysis of a large data set collected by the Digitalis Investigation Group as part of a multicentre trial. Their finding that women receiving digoxin were significantly more likely to die than women who received placebo can be considered robust because the 2 groups did not differ significantly at baseline with respect to important predictors of mortality such as age or cause or severity of disease. Men, on the other hand, were not more likely to die when treated with digoxin compared with placebo. When compared with men, the women in the study were older and had greater severity of HF and greater comorbidity. Although the researchers adjusted for these differences in their analysis, the data were derived from a trial where exploration of sex differences was not planned a priori. This resulted in a disproportionate number of men in the secondary analysis, which could be considered a possible limitation.

Women already account for most deaths from HF, and these study findings indicate that the risk is increased for women taking digoxin. Unfortunately, the study was unable to test explanations for the difference. However, it is worthwhile for nurses working with women with CHF to question the need for digoxin in the treatment plan. This study also supports the need for studies that incorporate stratification for sex in their design and execution.

Elizabeth Rideout, RN, PhD Associate Professor McMaster University Hamilton, Ontario, Canada

1 Hood WB Jr, Dans AL, Guyatt GH, et al. Digitalis for treatment of congestive heart failure in patients in sinus rhythm. Cochrane Database Syst Rev 2003;(1):CD002901. 